\title{
DUALITY THEORIES FOR METABELIAN LIE ALGEBRAS. II
}

BY

\author{
MICHAEL A. GAUGER
}

\begin{abstract}
In this paper I have replaced one of the axioms given in my Duality theory for metabelian Lie algebras (Trans. Amer. Math. Soc. 187 (1974), 89-102) concerning duality theories by a considerably more natural assumption which yields identical results-a uniqueness theorem.
\end{abstract}

1. Introduction. In [3] I defined and proved a uniqueness theorem for what I referred to as an algebraic duality theory for metabelian Lie algebras. In this paper I will investigate the duality theories which arise using axioms (I), (II), (III) and (V) of [3]. Axiom (V) was an easy consequence of the first four, and is really a more natural thing than (IV) to require of a duality theory. It will be shown that these weaker yet more natural axioms give rise to nearly identical results. To classify all metabelian Lie algebras $N$ such that $\operatorname{cod} N^{2}=g$ one must consider the cases where $0 \leqslant \operatorname{dim} N^{2} \leqslant\left(\begin{array}{c}g \\ 2\end{array}\right)$. A duality theory nearly cuts this problem in half in the sense that classifying those $N$ such that $\operatorname{dim} N^{2}=p$, is the same as classifying their duals $N_{D}$ which satisfy $\operatorname{dim} N_{D}^{2}=\left(\begin{array}{l}g \\ 2\end{array}\right)-p$. A duality theory shuffles among themselves those $N$ for which $\operatorname{dim} N^{2}=\left(\begin{array}{l}8 \\ 2\end{array}\right) / 2$, and apparently contributes nothing to their classification. Using axioms (I), (II), (III), and (V) for the definition of an algebraic duality theory, we prove

MAIN THEOREM. There is only one algebraic duality theory.

The assumption that the ground field $k$ is algebraically closed and of characteristic zero, together with the notation of $\S \S 1$ and 2 of [3], remain in force throughout this article. [2], [3] and [5] provide a suitable background.

Suppose $D_{1}, D_{2}$ are duality theories satisfying axioms (I), (II), (III) and (V) of [3] and let $V$ be a finite-dimensional $k$-vector space. Let $G_{p}\left(\wedge^{2} V\right)$ represent the projective Grassmann variety of all $p$-dimensional subspaces of $\Lambda^{2} V$. Consider $D_{1}, D_{2}: G_{p}\left(\bigwedge^{2} V\right) \rightarrow G_{m-p}\left(\bigwedge^{2}\left(V^{*}\right)\right)$ where $m=\operatorname{dim} \wedge^{2} V$. By (II), $D_{2}^{-1} \circ D_{1}=\rho$ is an automorphism of $G_{p}\left(\wedge^{2} V\right)$. We can assume $p \leqslant$ $\operatorname{dim} \wedge^{2} V / 2$ for otherwise we could consider $D_{1} \circ D_{2}^{-1}$. Suppose $S_{1}, S_{2} \in G_{p}\left(\wedge^{2} V\right)$ and $V \oplus \wedge^{2} V / S_{1} \cong V \oplus \wedge^{2} V / S_{2}$ (see $\S 1$ and Theorem 1 of [3]). Then by

Received by the editors July $24,1973$.

AMS (MOS) subject classifications (1970). Primary 17B30.

Copyright $\odot$ 1975, American Mathematical Society 
(V) for $D_{1}$ we have

$$
V^{*} \oplus \wedge^{2}\left(V^{*}\right) / D_{1}\left(S_{1}\right) \cong V^{*} \oplus \bigwedge^{2}\left(V^{*}\right) / D_{1}\left(S_{2}\right) .
$$

By (V) and (III) for $D_{2}$, we see that

$$
V \oplus \wedge^{2} V / D_{2}^{-1}\left(D_{1}\left(S_{1}\right)\right) \cong V \oplus \wedge^{2} V / D_{2}^{-1}\left(D_{1}\left(S_{2}\right)\right) \text {. }
$$

Thus, due to Theorem 1 of [3], the automorphism $\rho$ of $G_{p}\left(\wedge^{2} V\right)$ has the property that if $S_{1}, S_{2} \in G_{p}\left(\bigwedge^{2} V\right)$ and $S_{1} \equiv S_{2}(G L(V))$, then $\rho\left(S_{1}\right) \equiv$ $\rho\left(S_{2}\right)(G L(V))$. That is, $\rho$ induces a bijection of the quotient set $G_{p}\left(\wedge^{2} V\right) / G L(V)$. (Axiom (IV) of [3] had the effect of making $\rho$ centralize the image of $G L(V)$ in Aut $G_{p}\left(\bigwedge^{2} V\right)$. See $\S 1$ of [3].)

To establish a framework for the solution of this problem I wish to digress and consider the following situation. Let $G$ be an algebraic group acting on a variety $V$ by a morphism $G \times V \rightarrow U$. Then there is an induced map $G \rightarrow$ Aut $V$-the group of automorphisms of $V$. I wish to consider those automorphisms of $V$ compatible with the $G$-orbit structure. The set $V / G$ cannot always be given the structure of a variety in such a way that $V \rightarrow V / G$ is a morphism (in particular this would require orbits in $V$ to be closed; see [1]). Therefore, the best one seems to be able to ask in general is: what is the subgroup $S$ of Aut $V$ consisting of those automorphisms which induce bijections on $V / G$. $S$ is called the orbit shuffling group. Certainly $S \supseteq G$ (identifying $G$ with its image in Aut $V$ ), and sitting in between these two groups is the group $F$ which consists of those automorphisms stabilizing each orbit. $F$ is called the orbit fixing group. Thus $G \subseteq F \subseteq S$, and $F$ is normal in $S$ since it is the kernel of the homomorphism $S \rightarrow \operatorname{Bij}(V / G)$. In fact, $S$ is the normalizer of $F$ in Aut $V$. For this, suppose $\tau \in$ Aut $V$ normalizes $F$, and let $v_{1}, v_{2} \in V$ satisfy $\theta\left(v_{1}\right)=v_{2}$ for some $\theta \in G$. Then $\tau\left(v_{2}\right)=\tau \theta\left(v_{1}\right)=\theta^{\prime} \tau\left(v_{1}\right)$ for some $\theta^{\prime} \in F$. Since $\theta^{\prime}$ fixes $G$-orbits, there is a $\theta^{\prime \prime} \in G$ such that $\theta^{\prime}\left(\tau\left(v_{1}\right)\right)=\theta^{\prime \prime}\left(\tau\left(v_{1}\right)\right)$ and this completes the argument that $\tau \in S$.

Consider this situation for the action $G L(V) \times G_{p}\left(\bigwedge^{2} V\right) \rightarrow G_{p}\left(\bigwedge^{2} V\right)$ where $p \leqslant \operatorname{dim} \wedge^{2} V / 2$, and let $S, F$ be the orbit shuffling and fixing groups respectively. From the preceding discussion, the determination of the possible duality theories satisfying (I), (II), (III) and (V) of [3] for this particular $V$ and $p$ is precisely the problem of determining $F$ and $S$, each coset of $F$ in $S$ giving rise to a distinct theory in the following way. Let $\rho \in S$, let

$$
D: G_{p}\left(\bigwedge^{2} V\right) \rightarrow G_{m-p}\left(\bigwedge^{2}\left(V^{*}\right)\right), \quad D: G_{m-p}\left(\bigwedge^{2}\left(V^{*}\right)\right) \rightarrow G_{p}\left(\bigwedge^{2} V\right)
$$

( $m=\operatorname{dim} \bigwedge^{2} V$ ) be the Scheuneman-Gauger duality (see $\S 1$ of [3]). Then as $\rho$ runs over a set of distinct coset representatives of $F$ in $S$, the pairs 
$D \circ \rho: G_{p}\left(\wedge^{2} V\right) \rightarrow G_{m-p}\left(\wedge^{2}\left(V^{*}\right)\right), \quad \rho^{-1} \circ D: G_{m-p}\left(\wedge^{2}\left(V^{*}\right)\right) \rightarrow G_{p}\left(\wedge^{2} V\right)$

exhaust the possible theories for this $V$ and $p$. If two such theories for a $\rho_{1}$ and $\rho_{2}$ were to agree, that is,

$V^{*} \oplus \bigwedge^{2}\left(V^{*}\right) / D\left(\rho_{1}(S)\right) \cong V^{*} \oplus \bigwedge^{2}\left(V^{*}\right) / D\left(\rho_{2}(S)\right)$ for all $S \in G_{p}\left(\bigwedge^{2} V\right)$, then by a routine calculation (Theorem 1 of $\S 1$ of [3]) $\rho_{1}^{-1} \circ \rho_{2} \in F, \rho_{1} F=$ $\rho_{2} F$ which is impossible.

So let $V$ be a $g$-dimensional $k$-vector space and let $1 \leqslant p \leqslant\left(\begin{array}{l}g \\ 2\end{array}\right) / 2$. Consider the diagram

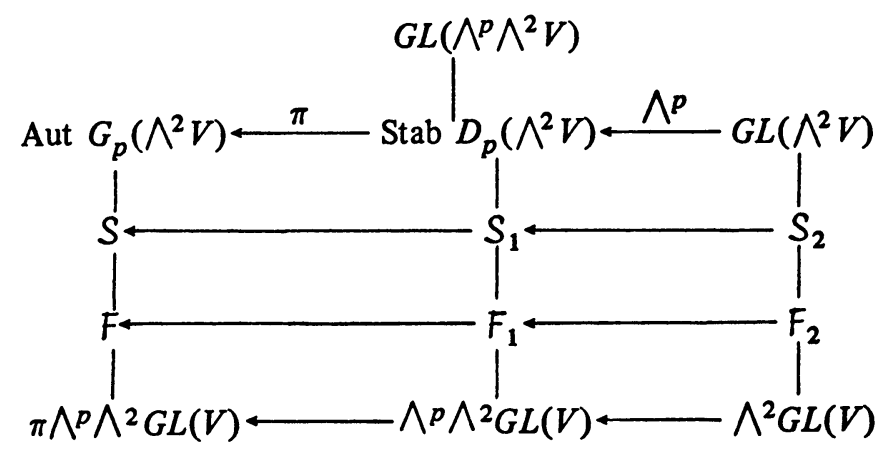

where $\pi$ is the natural map, Stab $D_{p}\left(\bigwedge^{2} V\right)$ is the stabilizer of the set $D_{p}\left(\wedge^{2} V\right)$ of decomposable $p$-vectors of $\bigwedge^{p} \bigwedge^{2} V, S_{1}=\pi^{-1}(S), F_{1}=\pi^{-1}(F), S_{2}=$ $\left(\wedge^{p}\right)^{-1}\left(S_{1}\right), F_{2}=\left(\bigwedge^{p}\right)^{-1}\left(F_{1}\right)$ and all other symbols have the same significance as in $\S \S 1$ and 2 of [3]. For the sake of simpler notation I have written $F_{2}, S_{2}$ instead of something like $F_{2, p}, S_{2, p}$ which would indicate more explicitly that a priori there is no reason to believe the $F_{2}$ 's and $S_{2}$ 's for different $p$ 's are related. By Proposition 3 of [3], $\pi$ is surjective. By Westwick's theorem (p. 1127 of [6, Theorem and the preceding remark]), $\wedge^{p} G L\left(\wedge^{2} V\right)=$ Stab $D_{p}\left(\bigwedge^{2} V\right)$ when $p<$ $\operatorname{dim} \bigwedge^{2} V / 2$, and when $p=\operatorname{dim} \bigwedge^{2} V / 2$ then $\left(\operatorname{Stab} D_{p}\left(\bigwedge^{2} V\right): \bigwedge^{p} G L\left(\bigwedge^{2} V\right)\right.$ ) $=2$. The determination of $F$ and $S$ is thus nearly always the same as determining $F_{2}$ and $S_{2} . S_{2}\left(F_{2}\right)$ is the group of linear transformations on $\wedge^{2} V$ shuffling (fixing) $G L(V)$-orbits of $p$-dimensional subspaces.

2. Determination of the orbit shuffling and fixing groups when $\operatorname{dim} V \neq 4$. Let $g=\operatorname{dim} V$ and suppose $g \neq 4$. We show first that $F_{2}=\bigwedge^{2} G L(V)$ relying heavily on Westwick's theorem $[6$, p. 1127] and the following algebrogeometric lemma whose proof was suggested to me by John Fogarty.

LEMMA 1. Let $W$ be an n-dimensional $k$-vector space and let $D \subset W$ be a d-dimensional homogeneous affine subset (not necessarily irreducible). For any 
$x \in W-D$ and any $p$ satisfying $1 \leqslant p \leqslant n-d$, there is a p-dimensional subspace $S$ containing $x$ such that $S \cap D=\{0\}$.

Proof. By the dimension of $D$ we mean the maximal dimension of its irreducible components. Go by induction on $n$. Let $H_{x}$ be the set of all hyperplanes of $W$ containing $x$, a closed irreducible subset of the Grassmannian of all hyperplanes of $W$. Write $D=D_{1} \cup \cdots \cup D_{m}$ where the $D_{i}$ are the irreducible components of $D$. For each $i$ it is easy to find a hyperplane containing $x$ but not all of $D_{i}$. Thus if we call $H_{x, i}$ the collection of all hyperplanes through $x$ but not containing $D_{i}$, we see that $H_{x, i}$ is a nonempty open subset of $H_{x}$. Hence $\bigcap_{i} H_{x, i}$ is nonempty, that is, there is a hyperplane $W_{1}$ through $x$ which contains none of the $D_{i}$. Then consider $W_{1}$ and $D^{\prime}=D \cap W_{1}$, and observe that $\operatorname{dim} D^{\prime} \leqslant d-1$ since each component of $D$ is reduced by the intersection with $W_{1}$. Apply the induction hypothesis to complete the proof.

Since the decomposable 2-vectors in $\Lambda^{2} V$ are a homogeneous affine subvariety of $\bigwedge^{2} V$ of dimension $2(g-2)+1$, we get the

COROllary 2. Let $x$ be a nondecomposable vector in $\wedge^{2} V$ and suppose $\operatorname{dim} V=g$. For any $p$ satisfying $1 \leqslant p \leqslant\left(\frac{g}{2}\right)-2 g+3$ there is a p-dimensional subspace $S$ containing $x$ but no nonzero decomposable vectors.

Proposition 3. If $1 \leqslant p \leqslant\left(\frac{g}{2}\right)-2 g+3$ and $g \neq 4$ then $F_{2}=\wedge^{2} G L(V)$.

Proof. Since $g \neq 4$, by Westwick's theorem $\left[6\right.$, p. 1127] $\wedge^{2} G L(V)$ is the stabilizer in $G L\left(\bigwedge^{2} V\right)$ of the set of all decomposable 2-vectors. Let $\rho \in F_{2}$, that is, $\rho$ stabilizes each $G L(V)$-orbit of $p$-dimensional subspaces. Suppose however that $\rho$ does not stabilize the set of decomposable 2-vectors in $\bigwedge^{2} V$. In particular, let $x$ be a decomposable vector in $\bigwedge^{2} V$ such that $\rho(x)$ is nondecom. posable. By Corollary 2 , we can find a $p$-dimensional subspace $S$ containing $\rho(x)$ but no nonzero decomposable. Now $\rho^{-1}(S)$ is a $p$-dimensional subspace containing $x$ and $\rho$ stabilizes $G L(V)$-orbits so there is a $\theta \in G L(V)$ with $\bigwedge^{2}(\theta)\left(\rho^{-1}(S)\right)=S$. This is clearly impossible since $\Lambda^{2}(\theta)$ takes decomposables to decomposables and $S$ has no nonzero decomposables. Hence $\rho$ stabilizes the set of decomposable vectors; this stabilizer is $\bigwedge^{2} G L(V)$.

Since we only need to pin down $F_{2}$ when $1 \leqslant p \leqslant\left(\frac{g}{2}\right) / 2$, we need to check when $\left(\begin{array}{l}g \\ 2\end{array}\right)-2 g+3 \geqslant\left[\left(\begin{array}{l}g \\ 2\end{array}\right) / 2\right]$ (i.e. greatest integer). An easy computation reveals this is the case whenever $g \geqslant 7$. Excluding $g=4$, the values of $g, p$ for which $F_{2}$ is not determined by Proposition 3 are: $g=3, p=1, g=5, p=4$ or 5 , and $g=6, p=7$.

I am forced to handle these remaining cases by a combination of ad hoc arguments which only serve to point out that if one was suitably good at Grassmannian geometry, a single proof for all cases could probably be given. 
If $\operatorname{dim} V=g=3$, then $\operatorname{dim} \bigwedge^{2} V=3$ and $G L\left(\bigwedge^{2} V\right)=\bigwedge^{2} G L(V)$. Thus $F_{2}=\bigwedge^{2} G L(V)$ when $g=3, p=1$.

Notice that if $\operatorname{dim} V=g$, there are subspaces $S$ of $\bigwedge^{2} V$ of dimension up to $(g-1)$ which consist entirely of decomposable vectors. Namely, they are subspaces of a subspace of the type $v \wedge V$ where $v$ runs over $V$. A subspace $v \wedge V$ will be called an $M_{\delta}$ subspace.

So suppose $g=5$ and $p=4$, and let $\rho \in F_{2}$. If $\rho$ does not preserve decomposables there is an $x \wedge y$ such that $\rho(x \wedge y)$ is not decomposable. By the paragraph above we can pick an $M_{\delta}$ subspace $S$ containing $x \wedge y$ and $\operatorname{dim} S=4$. Since $\rho \in F_{2}$ there is a $\theta \in G L(V)$ with $\Lambda^{2}(\theta)(S)=\rho(S)$. This is clearly impossible since $\Lambda^{2}(\theta)$ takes $M_{\delta}$ 's to $M_{\delta}$ 's and $\rho(S)$ is obviously not an $M_{\delta}$. Hence $\rho$ preserves decomposables. As before, this forces $F_{2}=$ $\bigwedge^{2} G L(V)$.

The remaining cases are handled using the following result.

LEMma 4. Let $S \subset \wedge^{2} V$ be an s-dimensional subspace of codimension at least two and containing no $M_{\delta}$ subspace. If $2(g-2)-s \geqslant 2$ where $g=$ $\operatorname{dim} V$, then there is an $(s+1)$-dimensional subspace $T$ containing $S$, and having no $M_{\delta}$ subspace.

Proof. An $M_{\delta}$ is $(g-1)$-dimensional. Let $v$ be any vector in $\wedge^{2} V-S$. If $S+\langle v\rangle$ has no $M_{\delta}$ subspace we are done. So suppose $S+\langle v\rangle \supseteq z \wedge V$ for some $z \in V$. Since $z \wedge V \nsubseteq S$ we know that $(z \wedge V) \cap S$ is $(g-2)$ dimensional. Let $w \in V$ be such that $z \wedge w \notin S$.

Now let $T$ be any other $(s+1)$-dimensional space containing $S$ and suppose it has an $M_{\delta}$ subspace $y \wedge V$. Then $(y \wedge V) \cap S$ is again $(g-2)$-dimensional, and since $2(g-2)-s \geqslant 2$, we have $(y \wedge V) \cap(z \wedge V) \equiv(y \wedge V)$ $\cap(z \wedge V) \cap S$ is at least 2-dimensional. That is, for some independent sets $\left\{z^{\prime}, z^{\prime \prime}\right\},\left\{y^{\prime}, y^{\prime \prime}\right\}$ in $V$, we have $z \wedge z^{\prime}=y \wedge y^{\prime}, z \wedge z^{\prime \prime}=y \wedge y^{\prime \prime}$. In terms of subspaces of $V$ these equations read $\left\langle z, z^{\prime}\right\rangle=\left\langle y, y^{\prime}\right\rangle,\left\langle z, z^{\prime \prime}\right\rangle=\left\langle y, y^{\prime \prime}\right\rangle$. Intersecting these results, we see that $\langle z\rangle=\langle y\rangle$ and $z \wedge V=y \wedge V$. In other words, the only $M_{\delta}$ in any $(s+1)$-dimensional subspace $T$ containing $S$, is $z \wedge V$. Since cod $S \geqslant 2$ we can enlarge $S$ to an $(s+1)$-dimensional space $T$ such that $z \wedge w \notin T$. Hence $z \wedge V \nsubseteq T$, and by preceding remarks this is sufficient to guarantee that $T$ has no $M_{\delta}$ subspace.

Now suppose $g=5, p=5$, or $g=6, p=7$. Let $\rho \in F_{2}$. If $\rho$ does not preserve decomposables there is an $M_{\delta}$ subspace $z \wedge V$ such that $\rho(z \wedge V)$ is not an $M_{\delta}$ subspace (in fact, since $\rho(z \wedge V)$ is $(g-1)$-dimensional, it contains no $M_{\delta}$ subspace). By one or two applications of Lemma 4 we can enlarge $\rho(z \wedge V)$ to a $p$-dimensional subspace $T$ containing no $M_{\delta}$ subspace. Set 
$S=\rho^{-1}(T)$ and note that $z \wedge V \subset S$. Since $\rho \in F_{2}$, there is a $\theta \in G L(V)$ satisfying $\Lambda^{2}(\theta)(S)=T$. But $\Lambda^{2}(\theta)$ takes $M_{\delta}$ 's to $M_{\delta}$ 's and $T$ contains no $M_{\delta}$ 's. This contradiction shows $\rho$ stabilizes the set of decomposable vectors and as before $F_{2}=\wedge^{2} G L(V)$. We have thus proven part of

Proposition 5. When $g \neq 4, F_{2}=\bigwedge^{2} G L(V)=S_{2}$.

Proof. The preceding lemma and discussion show that $F_{2}=\bigwedge^{2} G L(V)$. Every element of $S_{2}$ normalizes $F_{2}=\bigwedge^{2} G L(V)$. Considering the action of $\Lambda^{2} G L(V)$ on $\Lambda^{2} V$ this says that $S_{2}$ shuffles $\Lambda^{2} G L(V)$-orbits. There are exactly $[g / 2]+1$ such orbits (see $\S 5$ of $[2]$ ); $\{0\}, O_{1}, \cdots, O_{[g / 2]}$, and $O_{k}$ consists of those vectors which can be written as a sum of $k$-decomposables but not less than $k$. (Under the isomorphism $\bigwedge^{2} V \simeq \operatorname{Alt}\left(V^{*}\right)$ (§4 of [2]) the elements of $O_{k}$ correspond to forms of rank $2 k$ on $V^{*}$.) For each $i<j$, $O_{i} \subset \mathrm{Cl}\left(\mathrm{O}_{j}\right)$ where $\mathrm{Cl}$ denotes Zariski-closure. Thus, as varieties, the various $O_{i}$ have different dimensions. So if $\sigma \in S_{2}$ shuffles orbits, by a dimension argument it fixes them. In particular it fixes $\mathrm{O}_{1}$-the set of decomposable vectors. Westwick's result then forces $S_{2}=\bigwedge^{2} G L(V)$.

We are now prepared to prove

THEOREM 6. Suppose $g \neq 4$. If $p<\operatorname{dim} \wedge^{2} V / 2$ then $S=F=$ $\pi \bigwedge^{p} \bigwedge^{2}(G L(V))$. If $p=\operatorname{dim} \bigwedge^{2} V / 2$ then $\left(S: \pi \bigwedge^{p} \bigwedge^{2}(G L(V))\right) \leqslant 2$, hence $(S: F) \leqslant 2$.

Proof. If $p<\operatorname{dim} \Lambda^{2} V / 2$ then both $\pi$ and $\Lambda^{p}$ in the diagram at the end of $\S 1$ are surjective. So the result follows from Proposition 5. If $\operatorname{dim} \Lambda^{2} V / 2$ $=p$, then $\left(\operatorname{Stab} D_{p}\left(\bigwedge^{2} V\right): \Lambda^{p} G L\left(\bigwedge^{2} V\right)\right)=2$ by Westwick's result. Hence, since $\Lambda^{p}\left(S_{2}\right)=S_{1} \cap\left(\wedge^{p} G L\left(\Lambda^{2} V\right)\right)$, we get $\left(S_{1}: \Lambda^{p} S_{2}\right) \leqslant 2$. But $\wedge^{p}\left(S_{2}\right)=$ $\bigwedge^{p} \bigwedge^{2} G L(V)$, so $\left(S_{1}: \bigwedge^{p} \bigwedge^{2} G L(V)\right) \leqslant 2$. Now $\pi$ is always surjective, so in addition $\left(S: \pi \wedge^{p} \bigwedge^{2} G L(V)\right) \leqslant 2$, and obviously $(S: F) \leqslant 2$.

3. Determination of the orbit shuffling and fixing groups when $\operatorname{dim} V=4$. When $\operatorname{dim} V=4, \operatorname{dim} \wedge^{2} V=6$ and $p=1,2$, or 3 .

For the cases $p=1$ and $p=2$ we rely on the classification of the algebras $N$ such that $\operatorname{cod} N^{2}=4$ and $\operatorname{dim} N^{2}=5$ and 4 respectively ( $\S 5$ and Theorem 7.12 of [2]).

There are exactly two orbits in $G_{1}\left(\bigwedge^{2} V\right)$ when $\operatorname{dim} V=4$, the orbit $O_{1}$ of $\left\langle x_{1} \wedge x_{2}\right\rangle$ and the orbit $O_{2}$ of $\left\langle x_{1} \wedge x_{2}+x_{3} \wedge x_{4}\right\rangle$ where $x_{1}, \cdots, x_{4}$ is any basis of $V$. Now $\mathrm{O}_{1} \subset \mathrm{Cl}\left(\mathrm{O}_{2}\right)$ since the nondecomposable vectors in $\wedge^{2} V$ are open. Thus $\operatorname{dim} O_{1}<\operatorname{dim} O_{2}$. (The closure of an orbit 0 is the union of $\mathrm{O}$ and orbits of strictly smaller dimension.) Hence any automorphism of $G_{1}\left(\bigwedge^{2} V\right)$ which shuffles orbits must fix them. 
There are exactly three orbits in $G_{2}\left(\bigwedge^{2} V\right)$ when $\operatorname{dim} V=4$, the orbit $0_{1}$ of $\left\langle x_{1} \wedge x_{2}, x_{3} \wedge x_{4}\right\rangle$, the orbit $O_{2}$ of $\left\langle x_{1} \wedge x_{4}+x_{2} \wedge x_{3}, x_{2} \wedge x_{4}\right\rangle$ and the orbit $O_{3}$ of $\left\langle x_{2} \wedge x_{4}, x_{3} \wedge x_{4}\right\rangle$ where $x_{1}, \cdots, x_{4}$ is any basis of $V$. The dimensions of these three orbits are pairwise distinct. This can be shown by computing the ranks of the Jacobian at $1_{G L(V)}$ of the maps $\rho_{i}: G L(V) \rightarrow$ $\Lambda^{2} \wedge^{2} V$ where $\rho_{i}(g)=\Lambda^{2}\left(\Lambda^{2}(g)\right)\left(v_{i}\right)$ and $v_{1}=\left(x_{1} \wedge x_{2}\right) \wedge\left(x_{3} \wedge x_{4}\right), v_{2}=$ $\left(x_{1} \wedge x_{4}+x_{2} \wedge x_{3}\right) \wedge\left(x_{2} \wedge x_{4}\right), v_{3}=\left(x_{2} \wedge x_{4}\right) \wedge\left(x_{3} \wedge x_{4}\right)$ since $\operatorname{dim} O_{i}$ $=\left(\right.$ rank $\left.J \rho_{i}(1)\right)-1$. Since these computations are tiresome at best, I will indicate here a proof which is satisfactory when $k=\mathbf{C}$-the field of complex numbers. In this case any variety is also endowed with a strong topology (Chapter 1, $\S 10$ of [4]) and for any constructible subset of a variety, its closure in the strong topology is the same as its Zariski-closure. In particular, this holds for the orbits $\mathrm{O}_{1}, \mathrm{O}_{2}, \mathrm{O}_{3}$ since they are locally closed subvarieties [2, Lemma 7.7] of $G_{2}\left(\bigwedge^{2} V\right)$. Holding $x_{2}, x_{3}, x_{4}$ fixed and letting $x_{1}$ approach $x_{4}$, we see that $\mathrm{O}_{3}$ is contained in the strong closure of $\mathrm{O}_{1}$ hence $\mathrm{O}_{3} \subset \mathrm{Cl}\left(\mathrm{O}_{1}\right)$ where $\mathrm{Cl}$ is Zariski-closure. Similarly, since $\left\langle x_{2} \wedge x_{3}, x_{2} \wedge x_{4}\right\rangle$ is a representative of $O_{3}$, fixing $x_{2}, x_{3}, x_{4}$ and letting $x_{1}$ approach $x_{4}$ we see that $O_{3}$ is contained in the strong closure of $\mathrm{O}_{2}$, hence $\mathrm{O}_{3} \subset \mathrm{Cl}\left(\mathrm{O}_{2}\right)$. Thus $\operatorname{dim} \mathrm{O}_{3}<\operatorname{dim} \mathrm{O}_{1}$, $\operatorname{dim} \mathrm{O}_{2}$. Also $G_{2}\left(\wedge^{2} V\right)=\mathrm{O}_{1} \cup \mathrm{O}_{2} \cup \mathrm{O}_{3}=\mathrm{Cl}\left(\mathrm{O}_{1}\right) \cup \mathrm{Cl}\left(\mathrm{O}_{2}\right)$ is irreducible in the Zariski topology, so $G_{2}\left(\bigwedge^{2} V\right)=\mathrm{Cl}\left(\mathrm{O}_{1}\right)$ or $\mathrm{Cl}\left(\mathrm{O}_{2}\right)$. That is, either $\mathrm{O}_{1} \subset$ $\mathrm{Cl}\left(\mathrm{O}_{2}\right)$ or $\mathrm{O}_{2} \subset \mathrm{Cl}\left(\mathrm{O}_{1}\right)$. In either case $\operatorname{dim} \mathrm{O}_{2} \neq \operatorname{dim} \mathrm{O}_{1}$. Thus, since the orbits $\mathrm{O}_{1}, \mathrm{O}_{2}, \mathrm{O}_{3}$ have pairwise distinct dimensions, any automorphism of $G_{2}\left(\bigwedge^{2} V\right)$ which shuffles orbits must fix them.

We have thus seen that for $g=4$ and $p=1$ or 2 the groups $S$ and $F$ are identical. For the case $g=4, p=3$ we consider again the diagram of groups at the end of $\S 1$.

Since $\operatorname{dim} V=4, \wedge^{2} G L(V)$ is of index two in the stabilizer of the set of decomposable two-vectors-Stab $D_{2}$. So suppose $\rho \in F_{2}$ and $\rho$ does not preserve decomposables. Repeating the arguments of the case $g=5, p=4$ of $\S 2$ we arrive at a contradiction. So $\rho$ does stabilize decomposables. Hence $\left(F_{2}\right.$ : $\left.\wedge^{2} G L(V)\right) \leqslant 2$. But $\wedge^{2} G L(V)$ is connected, so it is the identity component of $F_{2}$. Now $S_{2}$ normalizes $F_{2}$, hence its identity component $\wedge^{2} G L(V)$. Repeating the arguments of Proposition 5, we see that $S_{2}$ preserves decomposables, thus $\left(S_{2}: \bigwedge^{2} G L(V)\right) \leqslant 2$. Since $3=\left(\begin{array}{l}4 \\ 2\end{array}\right) / 2, \bigwedge^{3} G L\left(\bigwedge^{2} V\right)$ is of index 2 in Stab $D_{3}\left(\wedge^{2} V\right)$, and thus $\left(S_{1}: \wedge^{p} S_{2}\right) \leqslant 2$. Hence

$$
\begin{aligned}
\left(S_{1}: \wedge^{p} \wedge^{2} G L(V)\right) & =\left(S_{1}: \wedge^{p} S_{2}\right)\left(\bigwedge^{p} S_{2}: \wedge^{p} \bigwedge^{2} G L(V)\right) \\
& \leqslant\left(S_{1}: \wedge^{p} S_{2}\right)\left(S_{2}: \bigwedge^{2} G L(V)\right) \leqslant 2 \cdot 2=4 .
\end{aligned}
$$


Now $\pi$ is always surjective so $\left(S: \pi \wedge^{p} \bigwedge^{2} G L(V)\right) \leqslant 4$. By the first and second main theorems of [3], however, $\left(F: \pi \wedge^{p} \wedge^{2} G L(V)\right) \geqslant 2$. That is, the automorphism $\rho$ of the first main theorem when $\operatorname{dim} V=4, p=3$ centralizes $\pi \wedge^{p} \wedge^{2} G L(V)$, hence shuffles orbits. But in the second main theorem it is shown $\rho$ fixes orbits. Thus $\rho \in F-\pi \wedge^{p} \wedge^{2} G L(V)$. Hence $(S: F) \leqslant 2$. We have thus proved

Theorem 7. Suppose $g=\operatorname{dim} V=4$. When $p=1$ or 2 then $S=F$, and when $p=3=\left(\begin{array}{l}g \\ 2\end{array}\right) / 2$ then $(S: F)<2$.

4. The uniqueness theorem. To establish the uniqueness it remains only to show $S=F$ when $p=\left(\begin{array}{l}g \\ 2\end{array}\right) / 2$. We know $(S: F) \leqslant 2$ in these cases, and in fact $(S: F)=\left(S_{1}: F_{1}\right)$ since $\pi$ is surjective and its kernel belongs to $F_{1}$. Now $S_{1}$ normalizes $F_{1}$, hence its identity component $\bigwedge^{p} \bigwedge^{2} G L(V)$. Likewise, since $\bigwedge^{p} \bigwedge^{2} S L(V)$ is the commutator subgroup of $\bigwedge^{p} \bigwedge^{2} G L(V), S_{1}$ normalizes it as well.

Suppose that $S_{1} \neq F_{1}$ and pick any $s$ in $S_{1}$. Now $s$ stabilizes $\wedge^{p} \wedge^{2} S L(V)$ by conjugation, so it stabilizes the corresponding Lie algebra $d\left(\Lambda^{p} \Lambda^{2}\right) s l(V)$ by conjugation $\left(d\left(\Lambda^{p} \bigwedge^{2}\right)\right.$ is the differential of $\left.\Lambda^{p} \bigwedge^{2}\right)$, inducing on it an automorphism. Now $d\left(\bigwedge^{p} \bigwedge^{2}\right)$ is an isomorphism of $\operatorname{sl}(V)$ onto $d\left(\bigwedge^{p} \bigwedge^{2}\right) \operatorname{sl}(V)$, and $\operatorname{sl}(V)$ has basically two types of automorphisms. The first are conjugations by elements of $G L(V)$, the second are compositions of a conjugation with the operation of taking a matrix to its negative transpose.

If the conjugation of $s$ on $d\left(\bigwedge^{p} \bigwedge^{2}\right) s l(V)$ is an automorphism of the first type, then multiplying $s$ by a suitable element of $\wedge^{p} \wedge^{2} G L(V)$ (only its coset matters) we can presume it centralizes $d\left(\Lambda^{P} \Lambda^{2}\right) s l(V)$, hence $d\left(\Lambda^{P} \Lambda^{2}\right) g l(V)$. The associative algebra spanned by $d\left(\wedge^{p} \bigwedge^{2}\right) g l(V)$ contains $\wedge^{p} \wedge^{2} G L(V)$, so $s$ centralizes it. By the first main theorem of [3] (second main theorem), when $g \neq 4(g=4)$, this contradicts $\left(S_{1}: F_{1}\right)=2$.

So suppose the conjugation of $s$ on $d\left(\wedge^{p} \Lambda^{2}\right) \operatorname{sl}(V)$ is of the second type. Multiplying $s$ by a suitable element of $\Lambda^{p} \wedge^{2} G L(V)$, and identifying $d\left(\wedge^{p} \wedge^{2}\right) s l(V)$ with $\operatorname{sl}(V)$, we can assume conjugation by $s$ induces - Id on the Cartan subalgebra $H$ of diagonal matrices of trace zero. Thus, as a linear transformation on $\wedge^{p} \wedge^{2} V, s$ must take any $H$-weight space to an $H$-weight space whose weight is the negative of the original one. Since the groups $S_{1}$ and $\wedge^{p} \wedge^{2} G L(V)$ have identical Lie algebras, and since the groups and their algebras stabilize the same subspaces of $\bigwedge^{p} \bigwedge^{2} V, s$ stabilizes the simple sl$(V)$-submodules. By the preceding remark, each of these simple submodules would have to be self-contragredient. If $\lambda_{1}, \cdots, \lambda_{l}$ are the fundamental weights of $s l(V)(\operatorname{dim} V=l+1)$, then the module contragredient to the simple $s l(V)$-module of highest weight $\Sigma_{i} m_{i} \lambda_{i}$ is the one with highest weight $\Sigma_{i} m_{l+1-i} \lambda_{i}$. Now applying the results 
of $\S 3$ of [3], one can pick out simple submodules of $\bigwedge^{p} \bigwedge^{2} V$ which are not self-contragredient. Thus this second type of automorphism cannot occur.

As a result $S_{1}=F_{1}$ for all $g, p$ and we have completed the proof of the

MAIN THEOREM. There is only one algebraic duality theory.

\section{REFERENCES}

1. J. Fogarty, Invariant theory, Benjamin, New York, 1969. MR 39 \#1458.

2. M. Gauger, On the classification of metabelian Lie algebras, Trans. Amer. Math. Soc. 179 (1973), 293-329.

3. -, Duality theories for metabelian Lie algebras, Trans. Amer. Math. Soc. 187 (1974), 89-102.

4. D. Mumford, Introduction to algebraic geometry, preliminary version, Harvard Univ. Press, Cambridge, Mass.

5. J. Scheuneman, Two-step nilpotent Lie algebras, J. Algebra 7 (1967), 152-159. MR 36 \#225.

6. R. Westwick, Linear transformations on Grassmann spaces, Pacific J. Math. 14 (1964), 1123-1127. MR 29 \#4766.

DEPARTMENT OF MATHEMATICS, UNIVERSITY OF MASSACHUSETTS, AMHERST, MASSACHUSETTS 01002 